\title{
EXPERIENCIAS DIDÁCTICAS DEL APRENDIZAJE EN LÍNEA EN LA UNIVERSIDAD DE MANAGUA: MEDIACIÓN CON LA TECNOLOGÍA
}

\author{
DIDACTIC EXPERIENCES OF ONLINE \\ LEARNING AT THE UNIVERSITY OF MANAGUA: \\ MEDIATION WITH TECHNOLOGY
}

Iliana Artiles Olivera ${ }^{1}$
https:// orcid.org/0000-0001-9882-6035

Dora María Meza Cornavaca ${ }^{2}$ https: / / orcid.org/0000-0002-6306-8382

Hazel Margarita Saravia García ${ }^{3}$ https://orcid.org/0000-0002-0624-6525

Ricardo Antonio Reyes Norori ${ }^{4}$ https:// orcid.org/0000-0002-8042-0173

Recibido: julio 11, 2020 - Aceptado: octubre 23, 2020

\section{RESUMEN}

La formación en línea es una necesidad prioritaria ante los nuevos requerimientos sociales; implica un profundo cambio de mentalidad entre los actores del proceso: capacidad de resolver problemas; asumir responsabilidades; cooperar; estimular el trabajo virtual en equipo; desarrollar la confianza en uno mismo; aprender a pensar de modo crítico e independiente; ser creativo e innovador y con iniciativa personal. El trabajo justifica la propuesta de una guía instruccional, que media la formación profesoral como ruta facilitadora del proceso de aprendizaje en línea para los estudiantes de la Universidad de Managua y la estimulación de estrategias de aprender a aprender, con el auxilio de la plataforma Edmodo. La investigación asume el enfoque cualitativo y para concebir la guía, se asume un proceso de intervención

1 Doctora en Ciencias Pedagógicas, Universidad Central «Marta Abreu», Cuba. Profesora del Departamento de Ciencias de la Información, Facultad de Matemática, Física y Computación, Universidad Central «Marta Abreu», Cuba. Miembro de la Red de Investigación de Aprendizaje iliao@uclv.edu.cu; ilianartiles2016@gmail.com

2 Ingeniero Industrial con estudios en Informática Empresarial, en Currículum y Docencia Universitaria, Universidad Latinoamericana de Ciencia y Tecnología de Costa Rica. Rectora de la Universidad de Managua (UdeM), Managua, Nicaragua. rectoría@udem.edu.ni

3 Arquitecta, Universidad Nacional de Ingeniería. Profesora de la Universidad de Managua, Nicaragua. hmsaravia 86@hotmail.com

4 Ingeniero en Computación, Universidad de Managua. Profesor de la Universidad de Managua, Nicaragua. ricardoreyes270491@gmail.com 
donde la vía inductiva orienta el curso de su construcción, a partir de las experiencias en que participaron investigadores, profesores y directivos, auxiliados de métodos y técnicas de corte cualitativo. Las actividades de aprendizaje implementadas para docentes, demuestran que se necesita de experiencias que combinen los saberes integrados, de apropiarse de herramientas y estrategias tecnológicas para encontrar información, crearla, reconstruirla y compartirla. De este accionar en los cursos y el diplomado, emerge el resultado: la guía instruccional, construida en la praxis investigativa, y que favorece la implementación de situaciones de enseñanza-aprendizaje metacognitivas para que el estudiante desarrolle capacidades, y gestione de manera independiente y autónoma su aprendizaje, mediado por la plataforma educativa Edmodo.

Palabras clave: aprendizaje, aprendizaje en línea, educación en línea, plataforma educativa, guía instruccional, experiencias didácticas, formación docente versátil.

\section{ABSTRACT}

Online training is a priority need in the face of new social requirements, which implies a profound change in mentality among the actors in the process: ability to solve problems, assume responsibilities, cooperate, stimulate virtual teamwork, develop self-confidence, learn to think critically and independently, be creative and innovative and with personal initiative. The work justifies the proposal of an instructional guide, which mediates teacher training as a facilitating route of the online learning process for students of the University of Managua and the stimulation of learning-to-learn strategies, with the help of the Edmodo platform. The research assumes the qualitative approach and to conceive the guide, an intervention process is assumed where the inductive path guides the course of its construction, based on the experiences in which the researchers, teachers and managers participated, aided by methods and techniques qualitative cut. The learning activities implemented for teachers show that experiences are needed that combine integrated knowledge, to appropriate tools and technological strategies to find information, create it, rebuild it and share it. From this action in the courses and diploma, the result emerges; the instructional guide, built on investigative praxis and that favors the implementation of metacognitive teaching-learning situations and that the student develops capacities to independently and autonomously manage their learning mediated by the Edmodo educational platform.

Key words: Learning, Online Learning, Online Education, Educational Platform, Instructional Guide, Didactic Experiences, Versatile Teacher Training.

\section{INTRODUCCIÓN}

En los momentos actuales se viven transformaciones en todos los ámbitos. Los avances de la ciencia y la tecnología resultan en un nuevo contexto social donde la Educación Superior está llamada a asumir los retos que le impone la sociedad de la información y el conocimiento.

Ante ellos, Delors (1996) enfatiza que la educación deberá modificar los aprendizajes, para lo cual promoverá saberes integrados: aprender a conocer, a hacer, a vivir juntos y a ser. En este sentido, la Educación Superior requiere convivir y apropiarse de los nuevos formatos de construcción del conocimiento y potenciar nuevas propuestas en la formación. 
En consecuencia, la formación inicial universitaria actual -que comprende un contexto social e histórico, caracterizado por la cultura digital donde emergen nuevos y variados modos de circulación de la información- trae aparejada, a la vez, nuevas modalidades en la construcción y representación del conocimiento por parte de los sujetos involucrados.

Desde esta perspectiva, el estudiante universitario tiene como reto fundamental la incorporación de nuevos conocimientos en su formación para potenciar su aprendizaje y realizarse como profesional. Según Ajofrín (2016), los retos son: autonomía, investigación, innovación y creatividad.

Asimismo, los avances de la tecnología de la información y la comunicación (TIC), han dado surgimiento a un modelo de aprendizaje: la educación en línea. Ante él, los docentes necesitan aprender multialfabetismos que favorezcan, en sus futuras prácticas de enseñanza, la generación de espacios para la construcción del saber en nuevos en tornos y formatos (Red Iberoamericana, 2012).

Procurando la mejora continua en torno a la educación en línea -auxiliada de plataformas educativas, así como las buenas prácticas que emergen de esta, en función del aprendizaje-, se acentúa la exigencia de mediar la formación profesoral con la propuesta de un instrumento, como ruta facilitadora del proceso de aprendizaje en línea, cuyo contenido se base en estrategias que promuevan la eficiencia en el proceso de aprender a aprender, en la modalidad en línea. En este sentido se refiere a la guía instruccional para el alcance del aprendizaje significativo mediado del facilitador.

En la Universidad de Managua (UdeM) se evidencia el trabajo académico, pero varios docentes -a partir de la supervisión continua desde la plataforma Edmodo-, muestran prácticas tradicionales en línea, homologando su quehacer con la modalidad presencial, queriendo dar salida al contenido como forma del saber y no al conocimiento en acción; este modo de enseñar ofrece una limitada oportunidad a la retroalimentación formativa y en consecuencia restringe, en los estudiantes, la posibilidad de aprender a aprender, es decir: limitando su propia concientización como aprendiz estratégico, condición esencial en el aprendizaje en línea.

No obstante, en la praxis existen experiencias satisfactorias que hacen necesaria la continuidad del accionar didáctico de formación en línea, buscando robustecer un proceso de enseñanza-aprendizaje para hacerlo más innovador, con estrategias que estimulen lo virtual con acompañamiento de un facilitador en la comunidad universitaria.

El presente trabajo responde a la exigencia de la estimulación del aprendizaje en línea en la UdeM y a la mediación con las tecnologías como experiencia didáctica, debido al interés que existe por la materia. Servirá para la activación del proceso de aprender a aprender en los estudiantes de las nueve carreras. El resultado de la investigación se aplica en todas las carreras de la universidad, usándolo como guía para organizar el propio aprendizaje, lo que conlleva un control eficaz del tiempo y la información. Se plantea así el siguiente objetivo general:

- Proponer una guía instruccional, que medie la formación profesoral como ruta facilitadora del proceso de aprendizaje en línea a los estudiantes de la Universidad de Managua, y la estimulación de estrategias de aprender a aprender, con el auxilio de la plataforma Edmodo. 


\section{ANTECEDENTES TEÓRICOS}

Diversas investigaciones explican la importancia de estimular el aprendizaje en los universitarios como algo novedoso. Se destacan, como relevantes en el contexto internacional, el estudio realizado por Ramírez (2012), experto en la temática, que marca las pautas de cómo activar y estimular el aprendizaje mediado por diferentes metodologías activas, o de aprender a aprender. De igual forma Monereo (1999), Arceo, Rojas y González (2002), Monereo y Badia (2013), plantean metodologías para diseñar casos profesionales que impulsen el autoaprendizaje.

La Comisión Europea enfatiza que la novedad en el aprendizaje se enmarca por la capacidad para proseguir y persistir, la conciencia de las necesidades y los procesos del propio aprendizaje, la identificación de las oportunidades disponibles, y la habilidad para superar los obstáculos con el fin de aprender con éxito (Ortega, 2008: 75).

De los análisis anteriores, se enfatiza que el aprendizaje consiste en aprender prácticas o experimentos, mediante la búsqueda y realización individual de la información; que depende de la voluntad, la iniciativa y la acción del que aprende, y que es el sujeto quien ha de reconocer las principales características de su propia lógica y auto dirección (Rivera, 2018).

En conferencia de prensa realizada a universitarios de la Universidad del Valle (2015), se afirma que el término «aprendizaje» se asocia al mundo académico, que es perdurable para toda la vida, que crea posibilidades al estudiante para su propia construcción, su autonomía personal, la capacidad de cambio y el ser un aprendiz activo, estratégico, reflexivo, cooperativo, responsable, mediante la activación del compromiso para pensar y una perspectiva de esperanza, a través del mantenimiento de la autorregulación cognitiva y afectiva de la actividad de aprender.

En este sentido, y asumiendo criterios autorales, estimular el aprendizaje tiene varias ventajas: fomenta la curiosidad, la investigación y la autodisciplina; se aprenden a resolver problemas por uno mismo; al no tener que seguir el ritmo de un grupo, puede dedicarse más tiempo en aquello que se dificulta y menos a lo que resulta más fácil; se es más constructivo; es una tarea que no solo permite confeccionar la habilidad, sino que ayuda a formar la personalidad positivamente (García, 1998).

Así, el hilo conductor se centra en que el aprendizaje es la tendencia para la mejora continua del proceso de enseñanza-aprendizaje al armonizar la función gestionadora del estudiante que aprende, pues este puede elaborar estrategias para aplicarlas en determinadas situaciones y así contribuir a un mejor aprendizaje por sí solo, condición importante para emprender los estudios universitarios en línea. Estos estudios son un desafío a nivel personal, para quien quiere demostrarse a sí mismo que puede alcanzar la meta (Butcher, 2015; García, 2017).

La educación en línea, representa una sorprendente opción para el aprendizaje y la formación académica, hoy necesaria para un mundo cada vez más competitivo. El incremento en la aplicación de las tecnologías de la información y las comunicaciones (TIC), facilita cada vez más el aprendizaje en línea como una opción accesible a la educación superior y a la práctica universitaria (Martínez 2009; Rodríguez, 2015; Sun y Chen, 2016). 
Estimular el aprendizaje en línea en los estudiantes -intencionarlo de manera flexible-, constituye una acción indispensable para que adquieran independencia y autonomía durante el aprender. Este reto implica aprovechar las funciones y los servicios de comunicación que proporcionan las TIC para instrumentar modelos didácticos basados en la flexibilidad y la autonomía del aprendizaje, facilitando el acceso a fuentes de información, canales de comunicación inmediata, interactividad, automatización de trabajos, pues es un proceso rápido y fiable de todo tipo de datos (Lewis y Spencer, 1986; Tarika, 2019).

Proveer aprendizaje en línea precisa el potenciar ámbitos de innovación educativa, auxiliados de una plataforma electrónica, que requiere del fomento de espacios colaborativos y al mismo tiempo del autoaprendizaje, para lo cual es necesario:

[...] proporcionar un problema interesante y real, la formación de equipos de trabajo reducido que participen en decisiones reflexivas, incentivar lecturas atractivas que insten a la problematización online, el desarrollo de habilidades comunicativas y aprendizaje auto dirigido y la propuesta de soluciones concretas partiendo del problema (Ramírez, 2012: 3).

En este accionar, y siguiendo las ideas del autor, el estudiante tiene un rol activo y responsable, abierto al trabajo en equipo, participativo, capaz de resolver problemas profesionales y de realizar búsqueda de información veraz que le ayude a solucionar problemas reales.

En este quehacer, el docente debe facilitar información; apoyar al estudiante en el desarrollo de habilidades de auto aprendizaje; diseñar la instrucción en línea con problemas interesantes, contextualizados y significativos, en función del perfil profesional y del aprendizaje activo; emplear recursos tecnológicos para hacer más asequible la situación didáctica que se desea, es decir ser el constructor de la experiencia y director del proceso hacia el aprendizaje (Mendoza, 2016).

En la educación en línea, el facilitador (profesor) con buenas prácticas, anima al estudiante a tomar contacto con la actividad; a cooperar entre sí; a tener un aprendizaje activo; a obtener una rápida realimentación; enfatiza el tiempo de la tarea para el autoaprendizaje; comunica elevadas expectativas y respeta los diversos caminos del aprendizaje (Pallof y Pratt, 2003; Vijil, 2016).

En resumen, debe ser un facilitador motivador; provocador de curiosidad intelectual y entusiasmo; dinamizador del trabajo colaborativo; gestor de actividades y entornos de aprendizaje que contemplen la diversidad de ritmos, estilos cognitivos, conocimientos y capacidades de los estudiantes, es decir, que resuelva dudas; promueva el uso de las TIC en diversos ambientes; transmisor de experiencia; evaluador de recursos y proveedor de los mismos a los estudiantes; co-aprendiz con los estudiantes, promoviendo un descubrimiento guiado e investigador que reflexione sobre la práctica y colabore con otros docentes facilitadores.

Asimismo, como plantea Ajofrín (2016), el facilitador potencia las actividades orientadas a: la movilización de saberes; ver prácticas sociales; descubrir nuevos saberes; plantear obstáculos; promover nuevos aprendizajes; permitir la identificación de logros y carencias; desarrollar la cooperación y la inteligencia; provocar la autoconfianza e identidad personal; promover la autonomía, y formar para la concepción y conducción de proyectos. En esta dinámica es transcendental que el estudiante ejerza un pensamiento crítico para analizar la realidad de su ámbito profesional, tomando en cuenta variados puntos de vista, conceptos, teorías, explicaciones y estrategias. 
Un punto de análisis importante y que emerge de las reflexiones anteriores, es la formación docente versátil como una opción inteligente, pues favorece la participación y la colaboración de todos e impulsa el trabajo colaborativo, tanto en el equipo docente, como con el entorno y entre los alumnos. Estimula el trabajo en equipo para ofrecer entornos de intercambio y discusión, participación activa en la toma de decisiones, espacios para reflexionar sobre lo que sucede en el aula virtual, lo cual significa romper con lo meramente presencial en un clima de aula virtual, donde el profesor dirige y decide, se motiva por auto aprender, comunica como una necesidad y desarrolla una cultura dinámica (Martín, 2018).

Desde esta perspectiva, se forma un docente con un perfil motivacional, cuya inspiración sobresaliente es la exploración, la contribución, la cooperación y el logro. Esto supone un equipo de docentes que trabajen en equipo, sean persistentes para conseguir los objetivos y mantengan un deseo de mejora e interés por aprender sin la necesidad de una compensación material; un grupo de interés clave con el fin de expandir las buenas prácticas hacia el cambio (Cummings y Worley, 2014, citado en Martín, 2018) y que mantenga además la motivación para el aprendizaje, la mejora y el logro (Cummings y Worley, 2014; Lenz, 2015, citado en Martín, 2018).

La formación docente versátil, estimula al equipo docente - paciente y flexible- para realizar cambios y adaptaciones (Kools y Stoll, 2016, citado en Martín, 2018) pues, en la modalidad virtual, debe emplear metodologías activas, fusionando factores y variables críticas en el aprendizaje. En concreto se incorpora: autovaloración del progreso de aprendizaje; discusión en el aula virtual y evaluación formativa; estrategias reflexivas; habilidades de estudio; tutorías por pares; ambiente agradable entre todos los actores, y gestión emocional que ofrece mayor autonomía al estudiantado (Hattie, 2012; Lenz, 2015, citado en Martín, 2018).

Otro interesante punto de análisis es la aplicación de estrategias de aprendizaje en línea, que sitúan al estudiante como máximo responsable de su proceso de aprendizaje, ya que es quien construye el conocimiento y nadie puede sustituirlo en esta tarea. La actividad constructiva del estudiante se aplica a contenidos que ya posee en un grado de elaboración considerable, es decir, la práctica de los contenidos que constituyen el núcleo de los aprendizajes escolares, son conocimientos y formas culturales (Otero, 2007: 199).

Vistas así, las estrategias de aprendizajes en línea -como el proceso de toma de decisión, consciente e intencional- consisten en seleccionar los conocimientos conceptuales, procedimentales y actitudinales para cumplimentar un determinado objetivo, siempre en función de la situación educativa en que se produce la acción (Monereo, 1999).

Las estrategias de aprendizaje en línea sugeridas son: estrategia decisoria (que el alumno emita juicios de valor acerca de la información y logre ser autocrítico), técnica de aprendizaje colaborativo (requiere que los equipos de trabajo se autorregulen en el desempeño de la actividad, como en las relaciones interpersonales) y macro reglas para el análisis de la lectura comprensiva (supresión, generalización, construcción e integración) (Alfina y Mendoza, 2020).

En síntesis, son varias las nuevas aplicaciones y propuestas educativas que orientan un cambio en los desafíos del estudiante para diseñar situaciones de aprendizaje con enfoque innovador, dirigido al aprendizaje en línea. Partiendo de este postulado, el docente facilitador requiere ser orientado y capacitado para comprender y aplicar en su praxis educativa en línea, las estrategias de autoaprendizaje basado en problemas, debate, argumentación y reflexión, vinculadas al aprendizaje con casos, las cuales le serán útiles para lograr, en sus clases virtuales, construcciones sociales del conocimiento hacia la generación de nuevas 
conclusiones y así desarrollar situaciones formativas innovadoras, mediadoras de la cultura y de los otros, para enriquecer las estructuras cognitivas y afectivas de los estudiantes y, por consiguiente, para estimular el proceso de aprender a aprender.

En concreto, los facilitadores deben dotarse de los marcos teórico-prácticos del neuroaprendizaje para diseñar, ejecutar y evaluar nuevas estrategias de enseñanza-aprendizaje; así mismo, tener ideas claras sobre las relaciones entre el conocimiento psicológico de los procesos de aprendizaje, la teoría y la práctica de una enseñanza estratégica, así como de la naturaleza sociocultural de los procesos de construcción del conocimiento en la mente humana; es decir, qué sentido psicológico tiene el comprender en el aprendizaje significativo y, por consiguiente, cómo desarrollar estrategias innovadoras para que el estudiante se convierta en un aprendiz estratégico (Mendoza, 2020: 10).

En consecuencia, el neuroaprendizaje es una disciplina que combina psicología, pedagogía y neurociencia para explicar cómo funciona el cerebro en los procesos de aprendizaje. Esto permite conocer los mecanismos para aprender tanto de la mente como de nuestro sistema nervioso, considerando los elementos y las condiciones óptimas para la estimulación del pensamiento crítico, creativo y metacognitivo de los educandos, así como el rol de los neuroeducadores (Loja, 2015).

Así, se acentúa la necesidad de mediar el trabajo profesoral con una propuesta de instrumento que ofrezca una ruta facilitadora para el logro del proceso de aprendizaje en línea de manera innovadora, equiparlos con una serie de estrategias para avanzar en la toma de control del proceso de aprender a aprender. Encaminada a este propósito, surge una guía instruccional para el alcance del aprendizaje en línea y su mediación con la plataforma educativa.

Las guías instruccionales sirven como cuerpo de fundamentación de conocimiento de una asignatura; proceso tecnológico que concreta, organiza y desarrolla, los distintos elementos de la situación de enseñanza-aprendizaje de cara a la consecución de una serie de objetivos. Estos diseños instruccionales deberán ser capaces de generar el conocimiento organizado y elaborado, indispensable para facilitar el procesamiento significativo de la información y el aprendizaje, y procurar que el estudiante desarrolle aquellas capacidades para gestionar de manera independiente y autónoma su aprendizaje (Martínez, 2009).

Además, Martínez (2009) enfatiza que las guías instruccionales deben diseñarse con el fin de dar mayor relevancia a los procesos antes que a los contenidos, y privilegiar actividades que los estudiantes deben realizar en interacción con sus compañeros en pequeños grupos de trabajo, con la comunidad o individualmente, pero siempre con la orientación del docente.

Los autores del trabajo, en correspondencia con las reflexiones anteriores, acentúan que la guía instruccional para el aprendizaje en línea y mediado por las plataformas educativas deben: centrar el proceso de aprendizaje en el estudiante; potenciar la colaboración permanente entre los propios estudiantes en trabajo en equipos y con el profesor; en las actividades, facilitar la aplicación de situaciones de la vida cotidiana; provocar la utilización de recursos de aprendizaje innovadores; motivar diferentes alternativas de evaluación formativa; ofrecer la posibilidad a múltiples respuestas y búsquedas de información variada y actualizada por parte de los estudiantes; estimular entornos virtuales para la expresión y la creación; favorecer el aprendizaje significativo, y estimular la curiosidad científica. 
La guía instruccional para el aprendizaje en línea, parte del programa de asignatura para, después, pasar a la secuencia lógica de la instrucción por medio de la elaboración de la planeación didáctica de la asignatura, que posteriormente se detalla de manera más completa a través de la elaboración del plan diario (UCA, 2020).

Las guías instruccionales son un material didáctico que debe orientar el aprendizaje en línea del estudiante. Ante su importancia como una estrategia de apoyo, se establecen los siguientes pasos para su elaboración, en un formato sencillo, flexible, que facilita de forma rápida su ejecución: datos generales (logo de la Universidad, tipo de actividad, asignatura y tema); introducción necesaria (importancia de la temática para su desarrollo profesional; conexión con los pre saberes e incluir elementos motivacionales que provoquen entusiasmo, interés y curiosidad por descubrir y aprender cada contenido programático de la asignatura; instrucción didáctica o actividades de aprendizaje (contenido, objetivos, actividades de aprendizaje, materiales de estudio, fechas de entrega de la tarea). Esta instrucción didáctica se identifica con el desarrollo de las actividades de autoaprendizaje de los contenidos programáticos de la asignatura, la cual debe realizarse con mayor detalle e instrumentos de evaluación.

Para la redacción de las actividades de aprendizaje es necesario: partir de un objetivo; definir la modalidad de participación (individual, grupal, pareja); clarificar el proceso a seguir para la realización de la actividad; indicar los recursos que utilizará el estudiante para llevar a cabo la actividad (mapa conceptual, infografía, murales, foro, presentaciones, cómic u otros); informar los límites temporales en los que se llevará a cabo la actividad; explicar la forma de envío, especificando el tipo de archivo que se quiere recibir (Word, Excel, pdf, ppt, etcétera), además del formato en que debe presentarse el trabajo solicitado (tipo de letra, tamaño de letra, justificado, interlineado, número de páginas...), e indicar el sistema de evaluación de la actividad, mediado por criterios evaluativos explícitos en un instrumento de evaluación (rúbrica, lista de cotejo, etcétera).

No es tarea fácil alcanzar un progreso hacia esquemas conceptuales y prácticos que sitúen el quehacer de la labor docente desde la perspectiva socioconstructivista en la modalidad en línea, sobre todo cuando en la praxis predominan esquemas teóricos tradicionales. Sin embargo, los docentes, están conscientes que una vez iniciada la propuesta de cambio para el logro de aprendices estratégicos (autoaprendizaje), el proceso de transformación continúa en su práctica profesional más allá de la culminación del trabajo.

\section{METODOLOGÍA}

Como vía para el desempeño profesional exitoso, y su comprensión en torno a la enseñanza en línea desde su rol, la formación continua del docente debe asumirse desde un paradigma cualitativo. Su empleo se justifica por la necesidad de «descubrir pautas, patrones y regularidades que facilitan la comprensión, el sentido, el significado, y la construcción personal y social que los sujetos mantienen en los contextos educativos en los que funcionan» (Barca, 1996: 19).

Partiendo de esta consideración, el enfoque cualitativo en el presente estudio deviene en una propuesta interpretativa orientada a describir e interpretar los fenómenos en su contexto natural para representar, detallada y profundamente, el comportamiento dinámico y global del proceso de formación del profesor en torno a la enseñanza en línea desde su rol. Al decir de Colas (1992), los estudios cualitativos son considerados un proceso activo, sistemático y 
riguroso de indagación dirigida, en el cual se toman decisiones sobre lo investigable, en tanto se está en el campo de estudio.

En coherencia con este enfoque y a partir de la necesidad de acompañar la formación del estudiante en línea, se establece una ruta de capacitación que inicia con el desarrollo de cursos y un diplomado a los docentes, referido a la didáctica innovadora en la educación superior, incidiendo principalmente en metodologías activas para la enseñanza aprendizaje, en educación mediada por tecnología; con apoyo de plataformas interactivas, desarrolladas en una espiral dialéctica buscando responder al objetivo descrito. Inmerso en esta ruta, está el diseño de una guía instruccional para el aprendizaje en línea.

Para la elaboración de la guía instruccional, se consideró la vía inductiva para determinar el curso de su construcción, orientada por un proceso de intervención durante el desarrollo de los cursos y el diplomado, que facilitaron la observación directa acerca del empleo del docente de las estrategias ejercitadas en las capacitaciones. Del propio proceso de intervención, en la socialización e intercambio dinámico entre los docentes y el formador, se fue construyendo la guía instruccional que guía el aprendizaje en línea.

La confiabilidad y la validez de la guía instruccional, se evidencia con los docentes durante la capacitación; ellos mismos la construyen y reconocen que los resultados alcanzados se aproximan a lo que ellos piensan y sienten, es decir, son verdaderos para ellos y para otras personas que han estado en contacto con el fenómeno estudiado. Además, muestra de su validez está en relación con las preocupaciones de la experiencia personal del profesor: una visión clara de su experiencia que a la vez permite eficientar su desempeño. De igual forma, se determinó la búsqueda y revisión de la literatura que normaliza la investigación respecto del proceso de búsqueda y registro, incluyendo: los términos que se establecieron para el estudio; las estrategias y fuentes de búsqueda; el registro de resultados; las normas de revisión; los criterios de inclusión y exclusión para las publicaciones examinadas, y la estrategia de síntesis de la información.

La formación continua del personal docente, constituye una estrategia sistemática en la institución, lo mismo que la inversión en su infraestructura tecnológica y la determinación de diseñar estrategias que abonen a la formación integral del estudiante, entre las que se evidencia el acompañamiento de su educación, auxiliada por tecnologías de información y comunicación. Es a partir de la necesidad de actualizar al personal docente, que surge una nueva demanda formativa justificada en preparar -teórica y metodológicamente- a docentes de nuevo ingreso y a la actualización del resto para la práctica de estrategias que potencian el aprender a aprender en él y extrapolarlo en su desempeño con el estudiante, y formar un aprendiz estratégico en la modalidad en línea, alcanzando con ello versatilidad en el proceso de formación.

Esta versatilidad permite a la institución dar una respuesta eficiente ante la pandemia global por el COVID-19 y frente a la necesidad de la enseñanza en línea.

La selección de los participantes, se basó en la experiencia docente en tecnologías educativas. Se logró el estudio exploratorio con 96 profesores, acompañados por cinco directivos como participantes en los cursos y el diplomado acordado.

En el tránsito de la investigación exploratoria para el cumplimiento del objetivo, se aplicaron métodos del nivel teórico y empírico de conocimiento, de naturaleza cualitativa, que facilitan el registro de metas de transformación docente, la observación participante, la entrevista cualitativa basada en conversaciones informales, la revisión documental y el grupo de discusión. 
Para la recolección y el análisis de la información se construyó una matriz de recogida y estudio de la información, comprendiendo las categorías del estudio y las capacitaciones directas, respaldadas por documentos actualizados y las propias experiencias pedagógicas del docente en formación que, de forma explícita, trataron el tema para dinamizar la praxis del profesor, según la base de la enseñanza en línea. Este artículo se construye a partir de los resultados obtenidos en la capacitación a los docentes; lo mismo que en la dinámica del grupo de discusión, la observación participante, la entrevista y la revisión documental.

\section{RESULTADOS}

De la triangulación metodológica de los métodos del nivel empírico aplicados -tomando en cuenta la caracterización del universo de profesores, en función de validar el desarrollo futuro de los mismos como individuos y grupo-, el pronóstico es favorable para desarrollar las capacidades necesarias para desempeñarse como profesor en la modalidad en línea. De este análisis emergen las siguientes potencialidades en la UdeM:

- Alto grado de inversión en infraestructura tecnológica, con laboratorios de computación que facilitan el espacio de encuentro para la formación docente.

- Reconocimiento de los directivos por la formación continua de sus docentes, orientada hacia prácticas innovadoras de enseñanza, coherentes con el modelo educativo, el contexto y la teoría socio-constructivista.

- Clima de confianza con tendencias humanistas en el colectivo pedagógico, demeritando resistencias al cambio.

- Formación previa en medios tecnológicos para la enseñanza aprendizaje.

- A nivel individual, la preparación teórica, metodológica y práctica sobre el contenido de la profesión del docente y en correspondencia con la carrera donde trabaja, desarrollo de cualidades facilitadoras en los profesores, como la aceptación, la empatía y el asertividad, implicación por desarrollar la modalidad en línea y una tendencia manifiesta hacia el mejoramiento de su desempeño profesional en la innovación educativa.

Durante el proceso de intervención pedagógica, y condicionado por las potencialidades antes descritas, se construyó un instrumento (anexo 1) que facilita la aplicación de las teorías y estrategias aprendidas en los cursos y diplomados sobre formación en línea, apoyado de metodologías activas e innovadoras. Comenzó con la presentación de los resultados al Consejo Universitario, los ejercicios realizados en el contexto de los cursos y el diplomado, los principales hallazgos, la concepción de la formación recibida y los aprendizajes adquiridos para facilitar la modalidad de estudio en línea, a partir de las experiencias didácticas experimentadas por los directivos y profesores de la UdeM, de un ejemplo de una guía instruccional y además de una actividad aportada por Mendoza (2020), donde se estimula el aprendizaje en línea con niveles de metacognición (anexo 2).

Las principales características de la guía instruccional se refieren a:

- La descripción de las capacidades y la experiencia docente con el propósito de que el estudiante conozca generalidades profesionales de su docente. 
- Que se realice una breve descripción de la asignatura, acotando su importancia en la práctica cotidiana o profesional, o ambas.

- Que el docente introduzca cada tema que imparte, oriente el trabajo y especifique fecha de entrega e interacción.

- Genere una rúbrica con el fin de que el estudiante tenga los referentes para realizar su trabajo.

A partir del análisis comprensivo del informe de investigación -resultado de la triangulación metodológica, emergida en el proceso de intervención de manera inductiva-, los participantes (directivos y profesores) aportan riqueza a la interpretación del estudio e instan a la estimulación del aprendizaje en línea, con experiencias didácticas innovadoras. Los principales criterios de mejora por parte de los docentes, se muestran a continuación:

- «Durante el proceso de formación logramos planear la asignatura en línea, cuyo primer eje de reflexión fue no incorporar en las actividades de aprendizaje el enciclopedismo, la prioridad de los contenidos, la inercia del estudiante y el papel preponderante del docente como transmisor de conocimientos».

- «Hoy, la UdeM necesita como institución educativa desarrollar los talentos y las capacidades de los estudiantes, a través de metodologías activas, una educación situada y contextualizada, promover el aprender-haciendo, el pensamiento analítico, crítico y el aprender a aprender, tanto de manera individual como con otros».

- «Los estudiantes precisan aprender a resolver problemas, a razonar sobre situaciones nuevas, a ser capaces de adaptarse a los cambios».

- «La modalidad en línea, facilita aprendizajes como placer en las actividades orientadas a los estudiantes, a la construcción y al descubrimiento, al desarrollo individualizado de capacidades metacognitivas y a la formación continua durante toda la vida».

- «El docente del siglo XXI en la modalidad en línea, debe motivar a los estudiantes en la formación de experiencias significativas, capaces de alterar los procesos neuronales, [experiencias] inspiradoras que hagan aflorar preguntas, interrogantes e hipótesis que respondan a cuestiones reales; a problemas con múltiples y flexibles soluciones y caminos; que les obliguen a interaccionar con otros y a crear equipo, haciendo más eficaz el aprendizaje en ellos».

- «Los textos, materiales y recursos orientados para el aprendizaje, deben estimular en los estudiantes el pensamiento científico, creativo, que enseñen a pensar, que vayan de lo conocido a lo novedoso, de lo simple a lo complejo, de lo directo a lo indirecto, de lo real a lo abstracto, que posibiliten un desarrollo gradual, sin frustraciones, por la imposibilidad de encontrar la solución».

- «La UdeM se ha preocupado y ocupado en lograr en los agentes educativos, buenas prácticas con el uso de la plataforma Edmodo, mediada por la capacitación continua a docentes y estudiantes hacia la construcción colectiva y/o colaborativa del conocimiento».

- «Hoy, yo ya logro elaborar e implementar guías de autoaprendizaje que potencien la autonomía del aprendizaje en mis estudiantes».

- «La plataforma Edmodo ya no se emplea como depositario de apuntes y documentos, sino para flexibilizar el proceso formativo de los estudiantes, realizar innovaciones educativas y transformar los procesos de evaluación».

Las expresiones anteriores y los aprendizajes adquiridos en el proceso de intervención, además de la búsqueda bibliográfica, favorecieron que el docente formado de la UdeM, sea un profesional: capaz de dialogar; un neuroeducador entendido en los conocimientos sobre cómo incentivar el aprendizaje en línea de manera activa; que diseñe nuevas técnicas para mejorar el aprendizaje, el desarrollo cognitivo y emocional de los estudiantes; que trabaje la 
motivación, la problematización y el clima áulico virtual, presentando problemas interesantes; que desarrolle una comunicación asertiva, favorable para que los estudiantes quieran y puedan hablar; que estimule la capacidad para autorregular su aprendizaje, para que se formulen preguntas, analicen críticamente, piensen creativamente, modelen cómo se hace algo; que establezcan conexiones con docentes y estudiantes; que transfieran conocimientos y visibilicen su aprendizaje; que muestren esfuerzo intelectual de manera continua, de tal modo que estimulen y desafíen el pensamiento en ellos.

\section{CONCLUSIONES}

El proceso de intervención pedagógica durante la impartición de los cursos y el diplomado permitió, a docentes y directivos, comprender el repertorio de modelos y estrategias que orientan la enseñanza en línea, y los procesos formativos que facilitan el diseño y la evaluación de situaciones de enseñanza-aprendizaje en ambientes innovadores, con el uso de la tecnología y la plataforma educativa Edmodo.

Las actividades de aprendizaje, orientadas por los formadores y desarrolladas por los profesores participantes, se direccionaron a demostrar que se necesita de experiencias que combinen los saberes integrados, de apropiarse de tecnologías, herramientas y estrategias para encontrar y almacenar información, crearla, reconstruirla y compartirla, siendo estas reflexiones las inspiradoras de la construcción conjunta de la guía instruccional para la estimulación del aprendizaje en línea en la UdeM.

La guía instruccional construida de manera inductiva y colaborativa para la estimulación del aprendizaje en línea, incita la práctica de situaciones de enseñanza-aprendizaje metacognitivas, y facilita que el estudiante desarrolle las capacidades para gestionar de manera independiente y autónoma su aprendizaje, mediadas por la plataforma educativa Edmodo $\mathrm{u}$ otros recursos tecnológicos.

La experiencia investigativa exploratoria amerita su continuidad en la UdeM, mediada por proyectos investigativos de mejoramiento profesional educativo, basado en aprendizajes experienciales, constatando la influencia formativa del desempeño de los profesores como aprendices estratégicos y autodidactas.

\section{ANEXO 1}

Estimado(a) profesor(a)

Le damos la bienvenida a la Educación en línea. Estamos seguros que esta experiencia será satisfactoria.

Expresarles que, para una exitosa implementación de los conceptos y herramientas de la educación en línea y semipresencial, todos los usuarios [profesor(a), estudiante, grupo, 
directivos], deben comprender y reconocer el inmenso cambio cultural que se está presentando en el proceso educativo, además el distanciamiento físico que recomienda la OMS y el Ministerio de Salud de Nicaragua ante la actual situación.

La educación ligada a los entornos virtuales, se sustenta en un arduo trabajo de continuo cambio y esfuerzo por parte del individuo, inmerso en la temática, para brindar la posibilidad de garantizar una didáctica que estimule la visión cognoscitiva y que despierte un interés igual o mejor que el ofrecido en un espacio educativo presencial, apoyado en herramientas visuales y auditivas, aprovechando el auge de la multimedia.

Enfatizamos de esta forma que la formación en línea favorece la participación activa de estudiantes y profesores(as), en un entorno que facilita la interacción, la evaluación y la cooperación entre iguales.

\section{El objetivo de la guía}

Aplicar la educación en línea en las asignaturas, auxiliado de la plataforma educativa Edmodo, como modelo que guía el proceso de enseñanza-aprendizaje.

A continuación, se ofrecen las pautas necesarias para emprender con la educación en línea en la UdeM en el II y III Cuatrimestre.

\section{Recomendaciones para preparar su clase virtual en la plataforma educativa Edmodo}

1. Su punto de partida es el calendario del día y desarrollo de asignación de los grupos, el programa analítico de la asignatura, la planeación didáctica, el documento «Primer día de clase», los materiales de estudio para la clase y la evaluación.

2. La metodología que implementará en línea cambia las funciones del docente y las del estudiante.

3. La comunicación se amplía y rompe barreras del tiempo.

4. La forma y el método de realizar la clase es compartido más allá del aula clase (pruebas, publicaciones, asignaciones, chat, mensajería pública y privada, lecturas actuales sobre el tema, vídeos demostrativos del profesor para realizar orientaciones y aclarar dudas, actividades individuales y colectivas, películas sobre el tema y retroalimentación oportuna vinculando el uso de YouTube y Conferencia TED para la visualización de documentales, vídeos conferencias), mediante enlaces sin necesidad de que el estudiante deba de descargar el archivo multimedia, además materiales pregrabado en vídeo mediado de aplicaciones como: Zoom, Screencast-o-matic, Camtasia Studio u otros.

\section{Paso a paso para preparar la asignatura en línea}

1. Indique a los estudiantes las normas que rigen la educación en línea, necesarias en la negociación pedagógica: vocabulario respetuoso, conducta adecuada, ética académica, trabajos originales (no plagio), retroalimentación para conocer debilidades, cumplimento de los plazos de entrega de asignaciones (tareas, actividades evaluativas), entrega 
de notas, nombre completo y correcto, número de carnet, foto de perfil actualizada tipo carnet, primer plano, vista de frente y haber aceptado la formación en línea en el entorno de la institución.

2. Publique el primer día de clase a los estudiantes, el día de la semana en el que recibirá los materiales de estudio, la asignación y cuando se hará la descarga de los trabajos realizados individual o grupal de la clase anterior (ver ejemplo 1). Recuerde siempre este calendario.

3. Trabaje en línea -auxiliado del programa de la asignatura- su planeación didáctica, el documento de presentación del primer día de clase (ver ejemplo 2), hoja de control de asistencia y evaluación (impresa o digital), materiales de estudio para cada clase y la evaluación por clase.

4. Organice las actividades a desarrollar en sus grupos de clases, para lo cual se le orienta la siguiente secuencia en la publicación en la plataforma como agenda semanal: saludo de bienvenida, orientación del contenido (temas), indicaciones para que el estudiante acceda a los recursos (adjuntando material de estudio), material de clase ubicado en la carpeta de Edmodo, pruebas y asignaciones (tareas) con su puntaje y tenga presente que la pantalla de Edmodo visto desde un móvil, el equivalente a Biblioteca, para el estudiante es Mochila.

5. Elabore la guía de autoaprendizaje (didáctica) con la siguiente estructura: tema, contenidos, objetivo(s), tiempo establecido para la actividad orientada, materiales o recursos necesarios, orientaciones para cada actividad, evaluación formativa o sumativa para cada actividad (rúbrica de evaluación).

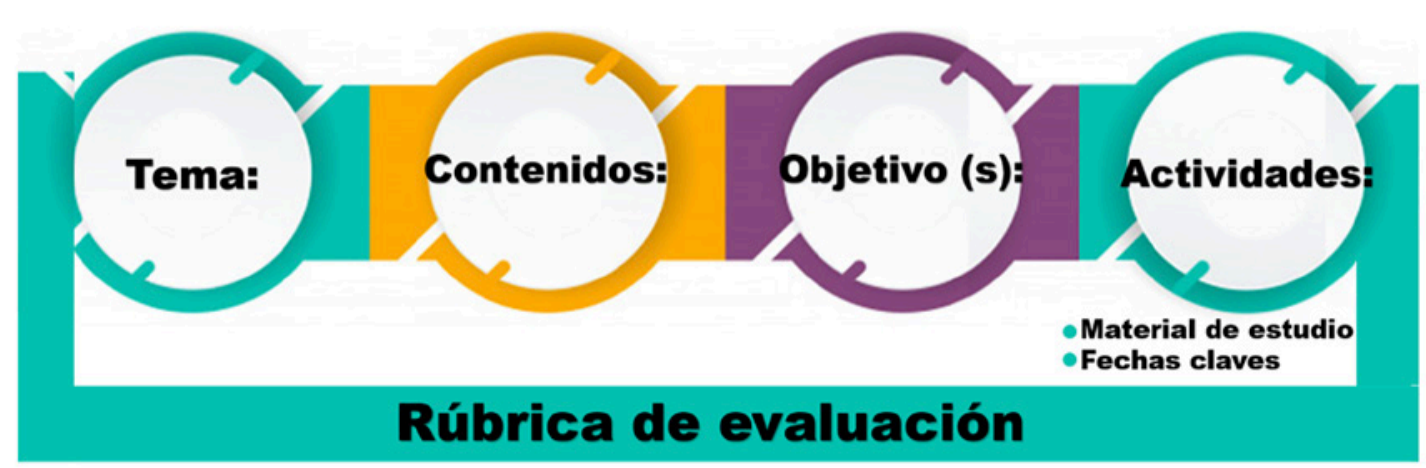

6. Retroalimente las actividades realizadas por los estudiantes, desde el puntaje obtenido hasta comentarios que le permitan saber en qué y cómo debe mejorar su aprendizaje, además es la justificación de la nota otorgada como evaluación (ver ejemplo 3). Incluir rúbricas para cada actividad y en caso oportuno hacer uso de formularios en línea para que el estudiante se autoevalúe.

7. Evalúe preferiblemente usando preguntas de verdadero y falso, selección múltiple o una combinación de ambas, también puede ser una asignación (trabajo). No se recomienda preguntas de completar y de unir.

8. Admita estudiantes que ingresen de forma tardía (previa revisión con registro) y oriéntelo para la revisión de las carpetas de estudio creadas en Edmodo para que se ponga 
al día con el contenido de las clases y sus asignaciones, el período será de una semana. Se les recomienda realizar revisiones periódicas en la pestaña Miembros de cada muro de clase (cada dos semanas) por las nuevas solicitudes de sus estudiantes, para la respectiva aprobación una vez comparada con el listado emitido por registro.

9. Mantenga una comunicación activa en la plataforma educativa Edmodo, en caso que los estudiantes presenten alguna duda. Para disminuir las dudas, es necesario que la guía práctica sea precisa y clara. Recuerde siempre al grupo de clase que las consultas deben enviarlas por mensajería privada al docente, identificándose con el nombre, grupo y asignatura a la que pertenece.

10. Realice búsqueda de los materiales de estudio a compartir con los estudiantes de acuerdo con la capacitación recibida para tal efecto. Estos pueden ser entre otros: libros, artículos, ensayos, resúmenes, manuales, monografía; siempre citando al autor o material de elaboración propia.

\section{Sugerencias de recursos didácticos}

1. Foro debate, infografía, portafolio electrónico, mapas conceptuales, línea de tiempo, murales digitales, cómics educativos, ensayo, relato, exposiciones fotográficas u otros.

2. Vídeo conferencia creada por el(la) profesor(a):

- Elija el formato más adecuado para el contenido a tratar, como diapositivas comentadas, entre otros (solicite ayuda a sus coordinadores; se sugiere visualizar el vídeo de cómo funciona Zoom, Screencast-o-matic y Screencast-o-matic).

- Cada vídeo debe tener una duración de hasta 15 minutos.

- El uso del vídeo es para el tratamiento de aspectos conceptuales necesarios, que puedan ocasionar dificultad o para la resolución de problemas que contengan gráficos, esquemas, etcétera. Importante, la primera clase amerita un vídeo sencillo y corto donde se presente el programa de la asignatura (presentación del profesor, objetivos generales de la asignatura, temas, principales contenidos por temas, formas de evaluación, formas de organización de las clases y utilidad práctica de la asignatura).

- Inserte en el vídeo, o la guía de 1 o 3 preguntas para la autoevaluación, además, scripts de esquemas y temas de debate.

- No mantener el mismo ritmo de la clase presencial, al ser un entorno en línea.

- Establecer encuentros por webconferencia al inicio de cada tema o semana, para la realización o seguimiento de una actividad significativa.

O Invitar al grupo de estudiantes que corresponde.

- Plantear actividades, incluyendo presentaciones, resolución de problemas orientados en la plataforma o directamente en la webconferencia que puedan comentarse posteriormente a través del chat, mensajería o dando voz y audio a los estudiantes. 
3. Empleo de YouTube y/o conferencias TED para la visualización de documentales, mediante enlaces sin necesidad de que el estudiante deba descargar el archivo multimedia (ver ejemplo 4).

\section{¡Éxitos en la preparación!}

Ejemplo 1. Calendario del día de publicación y descarga de asignación por grupos clase

\begin{tabular}{|c|c|c|c|c|c|c|}
\hline \multicolumn{7}{|c|}{ Docente: $\mathbf{x x x}$} \\
\hline $\begin{array}{l}\text { Código de } \\
\text { asignatura }\end{array}$ & Asignatura & \begin{tabular}{|l|} 
Código \\
Edmodo
\end{tabular} & $\begin{array}{l}\text { Publicación en muro } \\
\text { de clase y carpeta } \\
\text { (material de clase) }\end{array}$ & $\begin{array}{l}\text { Hora máxima de } \\
\text { publicación en } \\
\text { muro y carpeta }\end{array}$ & \begin{tabular}{|l} 
Descarga de tareas \\
y publicación de la \\
clase siguiente
\end{tabular} & $\begin{array}{l}\text { Hora máxima de } \\
\text { publicación y descargas } \\
\text { de tareas }\end{array}$ \\
\hline
\end{tabular}

Ejemplo 2. Documento presentación del primer día de clase

\section{1.- PRESENTACIÓN DEL DOCENTE}

a) Nombre y apellidos del docente:

b) Profesión y experiencias breves:

c) Lugar de trabajo:

d) Formas de comunicación:

$\checkmark$ Correo

$\checkmark$ Plataforma Edmodo

\section{2.- PRESENTACION DE LA ASIGNATURA}

a) Nombre de la asignatura:

b) Objetivo(s):

c) Utilidad práctica de la asignatura para el desarrollo de la profesión

c) Temas

d) Sistema de evaluación:

\section{3.- ORGANIZACIÓN DE LA CLASE}


Ejemplo 3. Ejemplo de cometarios de la revisión de una asignación

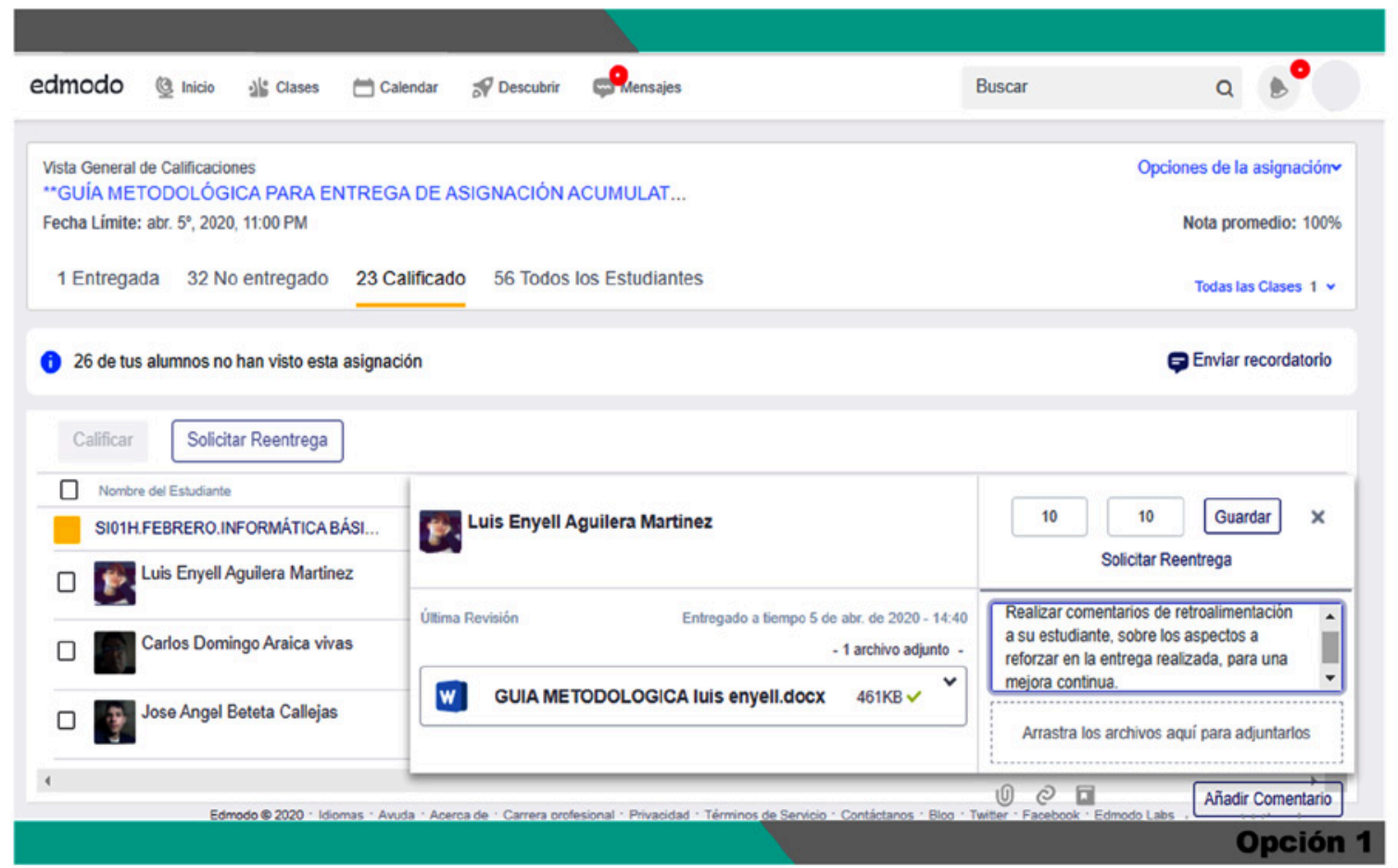

\section{Universidad de Managua}

Nombre: Yahaira Elizabeth Palacios Obando

Clase por suficiencia: Lectura crítica de textos periodisticos

Tarea \#1

FECHA: $31 / 07 / 2018$

Apreciada Yahaira.

Me gusta su trabajo. Recuerde que debe citar/referenciar el autor/fuente de donde toma la información,

Usted alcanzó un puntaje de $18 / 20$ puntos.

Saludos cordiales,

Lic. Ramirez

Que es una lectura? es el medio más ef ectivo para adquirir conocimientos, podemos tener muchos beneficios a través de este medio. Por ejemplo, la inteligencia, cuando un libro entra a nuestra cabeza inmediatamente nuestro nivel de imaginación se eleva, la sabiduria que el libro nos brinda se queda ahí para ser utilizada cuando más la necesitemos, cuando escuchamos decir a alguien que lee mucho inmediatamente nos parecer ser alguien culto, con mucho conocimiento.

Mejora la concentración. Con la cantidad de distracción que tenemos con la llegada de la era digital, hemos perdido la percepción de lo que es la concentración, si nos evaluamos. En cuestión de minutos nos distraemos ya sea revisando el celular o bien entrando directamente a las redes sociales o por cualquier motivo. La lectura sirve para ejercitar la concentración y disfrutar más del texto.

También mejora nuestra memoria. Cuando leemos una historia, libro, cuento etc. debemos memorizar los nombres de los protagonistas, sus historias y sus ambiciones. Absorbemos tanta información y datos nartirularoe nus cineornmn oiorririn nars la momnria

\section{Comentarios}

Oniversidad de Mana $02 \mathrm{c}$ Mejor si ¿Qué... (signo de interrogación e- es una pregunta?

Q Universidad de Managua lainformación. 


\section{Ejemplo 4. ¿Cómo situar enlaces para que el estudiante desde el} Edmodo visualice vídeos en You Tube?

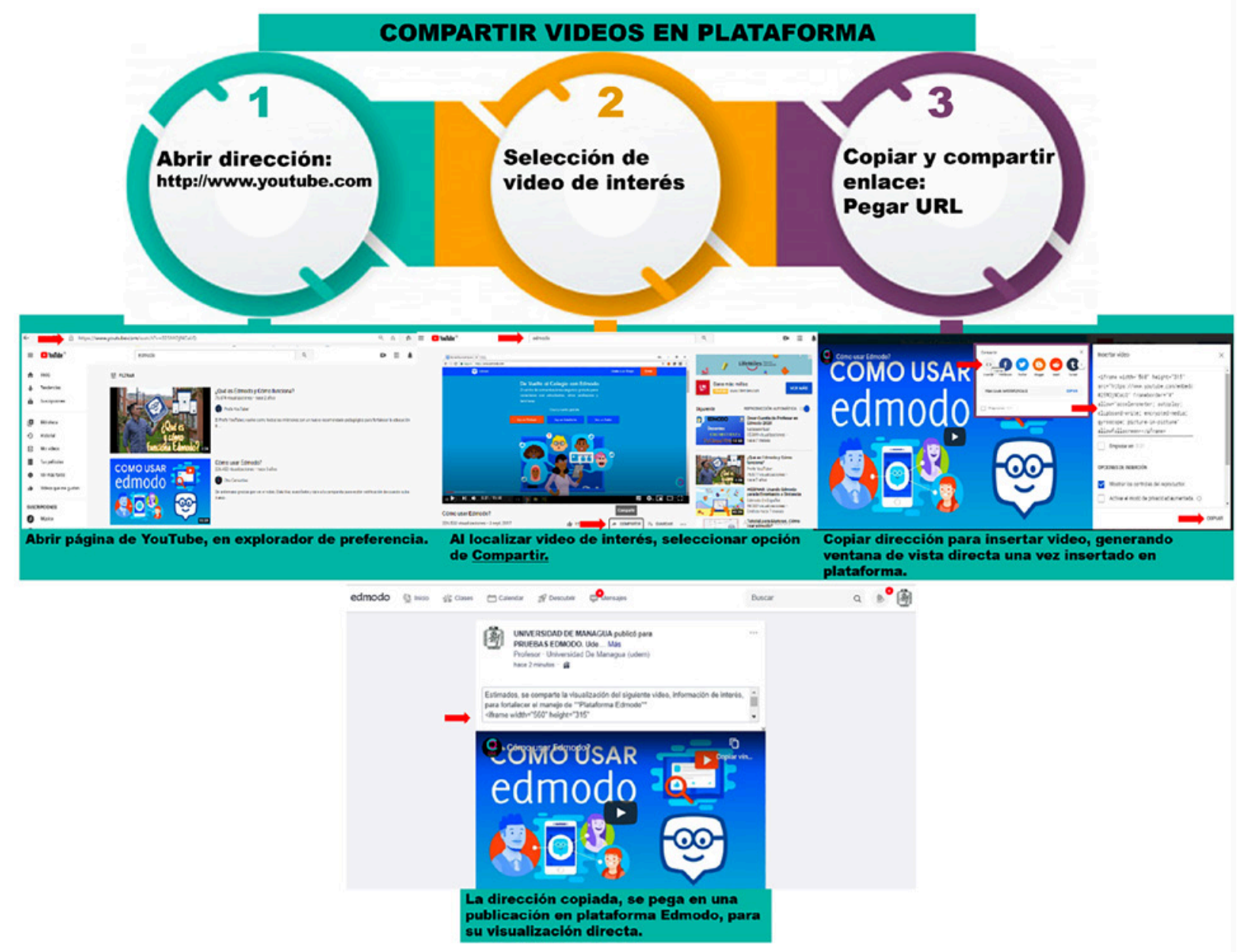

ANEXO 2

\section{PRÁCTICA DE INVESTIGACIÓN}

\section{Reporte de benchmarking: enfoques innovadores} en las instituciones educativas

El término «benchmarking» se define como el proceso de identificar productos, servicios y prácticas de los competidores, con el fin de incorporar las mejores prácticas a la propia empresa (Mendoza, 2020: 13).

Al tomar la educación superior este concepto y hacerlo suyo, la idea del benchmarking es precisamente la de ser un medio por el cual las instituciones educativas buscan conocer cuáles son las mejores prácticas educativas en el mundo y cómo los procesos de enseñanza-aprendizaje de diversos países contribuyen en mayor o en menor grado a lograr ciertas metas educativas.

Este ejercicio exige del equipo de trabajo el asumir y modelar el papel de funcionarios de una escuela, considerando que están buscando ejemplos de instituciones educativas que se caractericen por tener las mejores prácticas innovadoras. 


\section{Pasos}

\section{Actividad preparatoria}

a- El reporte de benchmarking se realizará con los mismos equipos de trabajo conformados en asignaturas anteriores.

b- Elegir un representante, líder o coordinador del grupo, responsable de subir a la plataforma Edmodo el reporte de benchmarking.

c- Es importante asegurar que toda la comunicación entre los miembros del equipo de trabajo se realice a través del foro de discusión asincrónica.

\section{Actividades de construcción documental}

Supóngase que ustedes tienen presupuesto suficiente para viajar alrededor del mundo y visitar distintas instituciones educativas. Antes de decidir a dónde viajarán, se imponen a sí mismos la tarea de hacer una especie de «tour virtual» en el que «visiten» algunas instituciones educativas a través de sus sitios web. Para comenzar el recorrido deberán:

a- Identificar un directorio de instituciones educativas en el mundo y definir el nivel educativo en el que trabajarán.

b- Seleccionar, de este directorio, cinco instituciones que representen al menos tres continentes (por ejemplo: dos de América, dos de Europa y una de Asia). Se solicita adjuntar el URL de las páginas web de estas instituciones.

c- Redactar una breve descripción de las cinco instituciones educativas seleccionadas, explicando bajo qué criterios se seleccionaron.

d- Definir el esquema de comparación de las distintas instituciones (los denominados «comparadores» o benchmarks).

e- Procesar los resultados en una matriz de comparación que muestre, en una columna, cada uno de los criterios de comparación, y en la otra, la columna de la matriz; colocar cada una de las cinco instituciones seleccionadas así como, en las celdas de intersección, la descripción de cada criterio comparativo.

f- Identificar las ideas innovadoras de las instituciones seleccionadas. Este punto es central en un estudio de benchmarking, en tanto exige que tengan una visión de líderes para hacer el proceso de «transferencia» de lo que se hace en otras instituciones educativas, y qué se puede y debe hacer en la suya; por consiguiente, se deberá justificar la razón por la que cada idea es valiosa.

g- Contrastar con la realidad de su centro. Se exige que asuman un papel de evaluadores/as, proponiendo conclusiones críticas sobre cómo las diferentes innovaciones de los centros internacionales visitados, pueden ser aplicadas al suyo a través de la propuesta de plan de mejora. 


\section{REFERENCIAS}

Ajofrín, L.G. (2016). Gigantes de la educación. Fundación Telefónic.

Alfina, O. (2020). Penerapan Ims-google classroom dalam pembelajaran daring selama pandemia Covid-19. Mmajalah Ilmiah Methoda, 10(1), 38-46.

Arceo, F.D.B., Rojas, G.H. y González, E.L.G. (2002). Estrategias docentes para un aprendizaje significativo: una interpretación constructivista. McGraw-Hill.

Barca, A. (1996). «Los métodos e investigación en la psicología de la instrucción». En J. Beltrán. Casablancas, S. y Odetti, V. (2017). Educación en línea: tendencias y nuevas experiencias. En https://www.flacso.org.ar/investigaciones/educacion-en-linea-tendencias-y-nuevas-experiencias/

Butcher, N. (2015). Guía Básica de Recursos Educativos Abiertos (REA). Francia: Organización de las Naciones Unidas para la Educación, la Ciencia y la Cultura (UNESCO).

Colas, M.P. (1992). Investigación educativa. Sevilla: Alfas. SA.

Cummings, T. \& Worley, C. (2014). Organization development and change. Cengage learning.

Delors, J. (1996.). «Los cuatro pilares de la educación». En La educación encierra un tesoro. Informe a la UNESCO de la Comisión Internacional sobre la Educación para el Siglo XXI. Madrid: Santillana/UNESCO.

García, C. (2017). Cómo funciona el aprendizaje en línea. En https:/ / revistaeducacionvirtual.com/archives/2876

García, M.R. (1998). Autoaprendizaje en la Educación Superior, Universidad Autónoma de Nuevo León. Documento impreso.

Hattie, J. (2012). Visible learning for teachers: Maximizing impact on learning. Nueva York: Routledge.

Kools, M. \& Stoll L. (2016). «What Makes a School a Learning Organisation?». OECD Education Working Papers, No. 137, OECD Publishing, Paris. En http://dx.doi.org/10.1787/5jlwm62b3bvh-en

Lenz, B. (2015). Transforming Schools Using Project-Based Learning, Performance Assessment, and Common Core Standards. San Francisco (CA): Jossey-Bass.

Loja, M. (2015). Neuroaprendizaje en el aula. Ecuador: ERIM.

Martín, D. (2018). La Organización educativa versátil y el desarrollo competencia. Tesis doctoral, Universidad Complutense, Madrid, España.

Martínez, A. C. (2009). «El diseño instruccional en la educación a distancia. Un acercamiento a los Modelos». Revista Apertura, 9(10), 104-119. 
Mendoza, A. (2016). El rol del docente universitario [archivo de vídeo]. En https:// youtu.be/IaEjxp0wQaU

Mendoza, C.A. (2020). Estrategias de Enseñanza-Aprendizaje. Diplomado: Desarrollo de capacidades pedagógicas. Facultad de Humanidades, Departamento de Educación, Universidad Centroamericana, Managua, Nicaragua.

Monereo, C. (1999). Aprender a aprender: una demanda de la educación del siglo XXI. Psicología de la instrucción 1. Variables y procesos básicos. Madrid: Síntesis.

Monereo, C. (1999). Concepciones sobre el concepto de Estrategias de Aprendizaje y sobre su enseñanza. Aula XXI. España: Santillana.

Monereo, C. y Badia, A. (2013). «Aprendizaje Estratégico y Tecnologías de la Información y Comunicación: Una revisión crítica. Teoría de la Educación». Educación y Cultura en la Sociedad de la Información, 14(2), 15- 41.

Ortega, E. M. (2008). «Aprender a aprender: clave para el aprendizaje a lo largo de la vida». Revista Participación Educativa, 9, 72-78.

Otero, I. et al. (2007). «Estrategias de Aprendizaje: del desarrollo intelectual al desarrollo integral». Acción Pedagógica, 16, 194-202.

Pallof, R. \& Pratt, K. (2003). The virtual student. San Francisco: Jossey Bass Wiley.

Ramírez, M.S. (2012). Modelos y estrategias de enseñanza para ambientes innovadores. México: Editorial digital. Tecnológico de Monterrey.

Red Iberoamericana (2012). Alfabetismos múltiples en la formación docente. Presentación Cyted 2012.

Rivera, B.A. (2018). Evidencias de los principios básicos del aprendizaje cooperativo. Documento impreso.

Rodríguez, R. (2015). Educar para la ciudadanía digital [archivo de vídeo].

Universidad Centro Americana (UCA), (2020). Paso a paso para elaborar la planificación de cursos virtuales y/o bimodales. Facultad de Humanidades y Comunicación. Departamento de Educación, Managua, Nicaragua.

Universidad del Valle, (2015). El autoaprendizaje método que toma fuerza en la Educación. Sala de Prensa.

Saavedra, M.A. (2001). «Aprendizaje basado en el cerebro». Revista de Psicología, X(1), 141-150.

Sun, A. \& Chen, X. (2016). «Online education and its effective practice: A research review». Technology Education, 15, 157-190.

Tarika, S.M. (2019). «Student's Perception Towards Effectiveness of E-Learning». International Bulletin of Management and Economics, X, 21-34.

Vijil, J. (2016). Los desafíos del docente universitario [archivo de vídeo]. En https:/ / youtu. be/tuMGlomnHTY 